Article

\title{
Performance Analysis of Interferometer Direction of Arrival Estimation under Frequency Mismatch of Array Manifold: DOA of Frequency Hopping Signal
}

\author{
Chan-Bin Ko and Joon-Ho Lee * \\ Department of Information and Communication Engineering, Sejong University, Seoul 05006, Korea; \\ bingo0217@sju.ac.kr \\ * Correspondence: joonhlee@sejong.ac.kr; Tel.: +82-2-3408-3195
}

Received: 30 January 2020; Accepted: 24 March 2020; Published: 29 March 2020

\begin{abstract}
We consider the direction of arrival (DOA) estimation of the frequency hopping (FH) signal. The frequency hopping $(\mathrm{FH})$ signal has been widely used for communication to control UAVs. Since the frequency of the FH signal is continuously changing, a mismatch may occur between the actual frequency of the received signal and the nominal frequency of the array manifold. In this paper, the azimuth and elevation estimation error in DOA estimation due to frequency mismatch are analytically derived. It is shown that the azimuth error is equal to zero and that elevation error depends on true elevation angle of the incident signal, rather than the true azimuth angle of the incident signal. The elevation error is also dependent on the actual frequency and the nominal frequency.
\end{abstract}

Keywords: frequency hopping; analytic error; interferometer; performance analysis; direction of arrival (DOA) estimation

\section{Introduction}

The direction of arrival (DOA) estimation of a signal source is important for electronic warfare. Detecting and monitoring the direction of high-speed vehicles such as UAVs (unmanned aerial vehicles) is essential to defend allies. Recently, high-speed UAVs have been developed for electronic warfare, because they can be used for attack or surveillance without human intervention [1,2].

Direction finding systems have wide applications in many military and civilian fields. In recent wars, electronic warfare has become important to defend against enemy attacks, both in aerial and underwater war. In aerial war, an active seeker could transmit and receive pulse signals and detect the DOA, velocity, and range of targets [3-5]. In an underwater war, the noise of a submarine propeller is used for localization. By using the spatially sparse property, high-resolution direction finding algorithms have been developed for underwater applications [6-8]. Recently, civilian fields for direction finding have been introduced for autonomous vehicles. Many sensors are required for self-driving. Especially, $77 \mathrm{GHz}$ radar sensors are used for detecting targets that are far away from radar sensors and for crash avoidance [9].

Frequency hopping signals could be used for wireless communication in UAVs. Using frequency hopping signals, information can be protected from various kinds of noise and multi-path distortion, and signal concealment and encryption are possible. Only the receiver having the spreading code can recover the encoded information [10]. DOA estimation of frequency hopping signals was studied in [11-14].

In frequency hopping $(\mathrm{FH})$ systems, the carrier frequency is continuously changing. In DOA estimation, strictly speaking, the frequency in the array manifold should be identically equal to the 
frequency of the incident signal. Since getting the instantaneous frequency of the incident FH signal is very hard to implement, nominal frequency should be used in the calculation of the array manifold. Therefore, there is a mismatch between the instantaneous frequency of the incident signal and the frequency in the array manifold, which results in the performance degradation of the DOA estimation algorithm.

In this paper, for the interferometer DOA estimation algorithm [15-17], explicit expressions of the azimuth estimation error and the elevation estimation error due to the frequency mismatch are derived and validated via numerical results.

In various previous studies, an analytic error due to phase measurement error for the interferometer DOA estimation algorithm was derived. Its correlation is defined by the difference between the measured phase of each sensor and phase of the array manifold of each sensor for all possible incident angles. The incident angle corresponding to the maximum value of correlation is chosen to be the DOA estimate [18-20]. In comparison with high-resolution algorithms like MUSIC [21] and ESPRIT [22], the interferometer algorithm is computationally efficient, but the performance in terms of the resolution and accuracy of the interferometer algorithm is worse than that of high-resolution algorithms. Various cost functions could be defined such as least squares, the cosine function, and a correlative method [23-25].

Differences in the cable length of the sensor array or thermal noise could generate an additive phase error for the phase measurement of the incident signal. The analytic error of the azimuth angle (1D DOA) of the signal incident on the uniform circular array has been derived [26], and the performances of the interferometer algorithms for various cost functions were compared in [27]. In [26] and [27], the authors derived an analytic expression of the error for the azimuth estimation of an interferometer algorithm.

In [27], the phase error was assumed to be a Gaussian random variable, and a uniform random variable was introduced to reflect the fact that the phase error could be any value between zero and $2 \pi$.

Therefore, the uniform distributed phase measurement error is more adequate for the case where the phase measurement error is due to random thermal noise. On the other hand, the Gaussian random variable tends to lie more densely around the mean values. Therefore, the Gaussian distributed phase error can be used for the phase error due to the difference in the RF cable lengths of the array system. Although the nominal lengths of the RF cable of the array system are equal, the actual lengths can be a little different from channel to channel.

Note that, in previous studies on the DOA estimation error due to phase measurement error, the possible frequency offset between the actual frequency of the incident signal and the nominal frequency used in the calculation of the array manifold was no explicitly taken into account.

In this paper, we are not concerned with performance degradation due to phase measurement error. That is, phase measurement error is not explicitly taken into account in this paper. On the other hand, we address degradation in the accuracy of the interferometer DOA estimation algorithm due to frequency mismatch or frequency offset between the actual frequency of an incident signal and the nominal frequency used for the array manifold.

Frequency offset or frequency mismatch occurs when the local oscillator signal for down conversion in the receiver cannot synchronize with the carrier signal contained in the received signal. This phenomenon can be attributed to two important factors: frequency mismatch in the transmitter and the receiver oscillators; and the Doppler effect as the transmitter or the receiver is moving. In direction of arrival estimation, an emitter transmitting the signal is not cooperative with the direction-finding (DF) station. Therefore, it is almost impossible to remove the frequency offset completely in DOA estimation.

A large frequency mismatch occurs in the DOA estimation of the frequency-hopping $(\mathrm{FH})$ signal, since the instantaneous frequency of an incident signal changes very rapidly, especially in a fast hopping environment. Frequency mismatch in this case is usually much larger than frequency 
mismatch due to failure in synchronization between the oscillator in the receiver and the oscillator in the transmitter.

In this paper, explicit expressions of the azimuth error and the elevation error are derived and validated using the numerical results. It is shown that the error obtained by simulations of the interferometer using a cosine function is equal to the analytically-derived error.

Our contribution in this manuscript does not lie in how much improvement can be achieved by proposing a new interferometer DOA algorithm or by modifying a previously existing interferometer algorithm. Our contribution in this paper lies in the analytic derivation of the azimuth estimation error and the elevation estimation error. It turns out that the azimuth estimation error is equal to zero and that the elevation estimation error can be obtained from the root of a second order equation or a fourth order equation depending on the approximations employed.

Our concern in this paper is how much the azimuth estimation error and elevation estimation error occur due to the mismatch between the frequency of an incident signal and the nominal frequency used for the array manifold. The phase at the $m^{\text {th }}$ antenna relative to that at the origin for an incident signal with a wavelength of $\lambda_{R F}$ from $\left(\theta^{(0)}, \psi^{(0)}\right)$ is defined as $\phi_{m}$, which is specified in (1) in Section 2. The corresponding quantity for a wave with a wavelength of $\lambda$, not $\lambda_{R F}$, from general direction $(\theta, \psi)$ is given by the first term in the brackets in (2) in Section 2.

We are concerned with, given $\left(\theta^{(0)}, \psi^{(0)}\right)$, what value of $(\theta, \psi)$ in $(2)$ in Section 2 maximizes the cost function in (2) when $\lambda_{R F}$ is different from $\lambda$. The arguments maximizing (2) are chosen to be the azimuth estimate and the elevation estimate. The difference between the azimuth estimate and the true azimuth is defined as the azimuth estimation error, and the difference between the elevation estimate and the true elevation is defined as the elevation estimation error.

The cost function in the interferometer DOA estimation algorithm can be defined in several ways. In this paper, we adopt the cosine-based cost function [26]. The square error-based cost function can also be used [27]. Note that maximizing the cost function is required in the cosine-based cost function and that minimizing the cost function is required in the square error-based cost function

By using a two-dimensional array, the azimuth and the elevation of an incident signal can be estimated simultaneously. Indeed, if the frequency hopping signal is wide-band, algorithms such as CSSM (coherent signal subspace method) should be used for DOA estimation [28-32]. The wide-band DOA estimation algorithm was introduced because the frequency of the array manifold differs from the carrier frequency of the signal source. Actually, the wide-band frequency of the signal source is focused and converted to manifold frequency in the CSSM algorithm. In practice, it is effective to use the narrow-band algorithm if possible, because it requires a large amount of computation to implement a wide-band direction-finding algorithm. In addition, the wide-band DOA algorithm requires an initial guess of the DOA estimate.

Analysis of the azimuth error and the elevation error due to frequency mismatch is presented in this paper. From the viewpoint of the CSSM algorithm, this study examines how the performance of the DOA estimation algorithms degrades when the frequency of an incident signal is not exactly equal to the nominal frequency used for the calculation of the array manifold.

In Section 2, we propose a method to derive the expressions of the azimuth estimation error and the elevation estimation error analytically in the presence of the frequency mismatch between the frequency of an incident signal and the nominal frequency used for the calculation of the array manifold. A summary of the derivation in Section 2 is presented in Section 3. In Section 4, the analytically-derived estimation errors are compared with the simulation-based estimation errors to validate the derived expressions of the azimuth and the elevation error. Section 5 concludes the paper.

\section{Derivation of the Azimuth and the Elevation Error under Frequency Mismatch}

Figure 1 illustrates the geometry of an interferometer direction of arrival estimation where $\mathbf{z}_{m}$ denotes the $m^{\text {th }}$ sensor location and $\kappa$ denotes a direction vector for an incident signal from $\theta=\theta^{(0)}$ and $\psi=\psi^{(0)}$. A two-dimensional array is adopted for simultaneous estimation of the azimuth and 
elevation. For a uniform circular array (UCA) with $M$ antennas, the expression in (1) represents the phase difference between the phase at the $m^{\text {th }}$ sensor location and the phase at the origin for an incident signal from $\theta=\theta^{(0)}$ and $\psi=\psi^{(0)}$ :

$$
\varphi_{m}=\frac{2 \pi r}{\lambda_{R F}} \cos \left(\theta^{(0)}-\frac{2 \pi m}{M}\right) \cos \left(\psi^{(0)}\right) .
$$

where $\lambda_{R F}$ denotes the wavelength of an incident signal.

The derivation of (1) is given in Appendix A. In (1), $\theta^{(0)}$ and $\psi^{(0)}$ are the true azimuth and true elevation. $r$ is the radius of an array antenna system. $M$ is the number of sensors.

In the interferometer algorithm using the cosine cost function, the cost function [16] can be written as:

$$
J(\theta, \psi)=\sum_{m=0}^{M-1} \cos \left[\frac{2 \pi r}{\lambda} \cos \left(\theta-\frac{2 \pi m}{M}\right) \cos (\psi)-\frac{2 \pi r}{\lambda_{R F}} \cos \left(\theta^{(0)}-\frac{2 \pi m}{M}\right) \cos \left(\psi^{(0)}\right)\right] .
$$

The expression in the brackets of Equation (2) is the phase difference between two phase values. The former phase expression in the brackets of Equation (2) is the relative phase at the $m^{\text {th }}$ sensor location with respect to the phase at the origin for the incident signal from azimuth $=\theta$ and elevation $=\psi$ associated with the wavelength of $\lambda$.

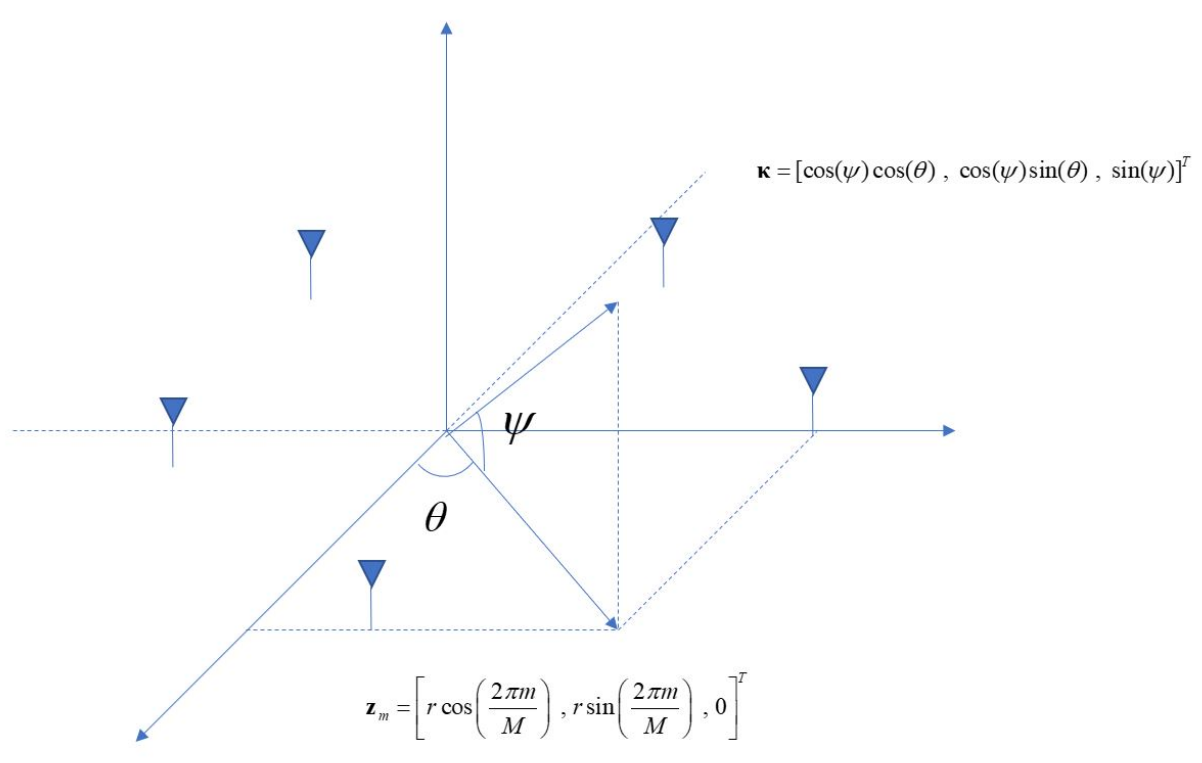

Figure 1. Sensor locations of the uniform circular array (UCA) and direction vector for an incident signal.

The latter phase expression in the brackets of Equation (2) is the relative phase at the $m^{\text {th }}$ sensor location with respect to the phase at the origin for the incident signal from the azimuth $=\theta^{(0)}$ and elevation $=\psi^{(0)}$.

Since $\cos (x)$ has a local maximum at $x=0$ for $-\pi \leq x \leq \pi$, the cost function is maximized when the two phase expressions in the brackets are equal. Therefore, we are concerned with what values of $\theta$ and $\psi$ in the former phase expression maximize the expression in (2), which is equivalent to what values of $\theta$ and $\psi$ in the former phase expression make the former phase value equal to the latter phase value. 
For the cost function in (2) to be maximized at $\theta=\theta^{(0)}+\Delta \theta$ and $\psi=\psi^{(0)}+\Delta \psi$, the partial derivative of the cost function in (2) with respect to the azimuth, $\theta$, and the partial derivative of the cost function in (2) with respect to the elevation, $\psi$, should be simultaneously zero at $\theta=\theta^{(0)}+\Delta \theta$, $\psi=\psi^{(0)}+\Delta \psi:$

$$
\begin{gathered}
\frac{\partial J(\theta, \psi)}{\partial \theta} \mid \begin{array}{c}
\theta=\theta^{(0)}+\Delta \theta \quad=0 . \\
\psi=\psi^{(0)}+\Delta \psi
\end{array} \\
\frac{\partial J(\theta, \psi)}{\partial \psi} \mid \begin{array}{c}
\theta=\theta^{(0)}+\Delta \theta \\
\psi=\psi^{(0)}+\Delta \psi
\end{array} \quad=0
\end{gathered}
$$

In this section, it is rigorously derived how $\Delta \theta$ and $\Delta \psi$ can be obtained from the constraints of (3) and (4). Note that, $\Delta \theta$ and $\Delta \psi$ are the azimuth estimation error and the elevation estimation error, respectively. Therefore, the final objective of this paper is to get expressions of the azimuth error and the elevation error without actually maximizing the cost function in (2) via computationally intensive exhaustive two-dimensional search with respect to $\theta$ and $\psi$.

\subsection{Partial Derivative of the Cost Function with Respect to the Azimuth} by:

The partial derivative of the cost function, $J(\theta, \psi)$, in (2) with respect to the azimuth, $\theta$, is given

$$
\frac{\partial J(\theta, \psi)}{\partial \theta}=\sum_{m=0}^{M-1}\left[\begin{array}{c}
\sin \left(\frac{2 \pi r}{\lambda} \cos \left(\theta-\frac{2 \pi m}{M}\right) \cos (\psi)-\frac{2 \pi r}{\lambda_{R F}} \cos \left(\theta^{(0)}-\frac{2 \pi m}{M}\right) \cos \left(\psi^{(0)}\right)\right) \\
\cdot \frac{2 \pi r}{\lambda} \sin \left(\theta-\frac{2 \pi m}{M}\right) \cos (\psi)
\end{array}\right]
$$

The first necessary condition for $\theta=\theta^{(0)}+\Delta \theta, \psi=\psi^{(0)}+\Delta \psi$ to be a critical point of the cost function $J(\theta, \psi)$ is that the partial derivative of the cost function, $J(\theta, \psi)$, in (2) with respect to the azimuth, $\theta$, should be identically zero at $\theta=\theta^{(0)}+\Delta \theta, \psi=\psi^{(0)}+\Delta \psi$ :

$$
\left.\frac{\partial J(\theta, \psi)}{\partial \theta}\right|_{\begin{array}{c}
\theta=\theta^{(0)}+\Delta \theta \\
\psi=\psi^{(0)}+\Delta \psi
\end{array}}=\sum_{m=0}^{M-1}\left[\begin{array}{c}
\sin \left(\frac{2 \pi r}{\lambda} \cos \left(\theta^{(0)}+\Delta \theta-\frac{2 \pi m}{M}\right) \cos \left(\psi^{(0)}+\Delta \psi\right)-\frac{2 \pi r}{\lambda_{R F}} \cos \left(\theta^{(0)}-\frac{2 \pi m}{M}\right) \cos \left(\psi^{(0)}\right)\right) \\
\cdot \frac{2 \pi r}{\lambda} \sin \left(\theta^{(0)}+\Delta \theta-\frac{2 \pi m}{M}\right) \cos \left(\psi^{(0)}+\Delta \psi\right)
\end{array}\right]=0 .
$$

Since $\sin (\phi)$ is approximately equal to $\phi$ for small $\phi$, (6) can be written as:

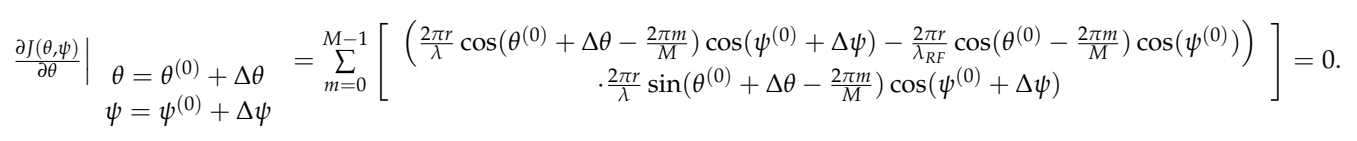

Let $a_{m}$ be defined as $\theta^{(0)}-\frac{2 \pi m}{M}$. Dividing both sides of $(7)$ by $\frac{(2 \pi r)^{2}}{\lambda}$ yields:

$$
\sum_{m=0}^{M-1}\left[\left(\begin{array}{l}
\frac{1}{\lambda} \cos \left(a_{m}+\Delta \theta\right) \cos \left(\psi^{(0)}+\Delta \psi\right)\left(\sin \left(a_{m}+\Delta \theta\right) \cos \left(\psi^{(0)}+\Delta \psi\right)\right) \\
-\frac{1}{\lambda_{R F}} \cos \left(a_{m}\right) \cos \left(\psi^{(0)}\right) \cdot\left(\sin \left(a_{m}+\Delta \theta\right) \cos \left(\psi^{(0)}+\Delta \psi\right)\right)
\end{array}\right)\right]=0 .
$$

From the double angle formula for the sine function, (8) can be written as:

$$
\sum_{m=0}^{M-1}\left[\left(\begin{array}{l}
\frac{1}{\lambda}\left(\frac{1}{2} \sin \left(2 a_{m}+2 \Delta \theta\right) \cos ^{2}\left(\psi^{(0)}+\Delta \psi\right)\right) \\
-\frac{1}{\lambda_{R F}} \cos \left(a_{m}\right) \cos \left(\psi^{(0)}\right) \cdot\left(\sin \left(a_{m}+\Delta \theta\right) \cos \left(\psi^{(0)}+\Delta \psi\right)\right)
\end{array}\right)\right]=0 .
$$

Applying the angle sum identity for the sine function results in: 


$$
\sum_{m=0}^{M-1}\left[\left(\begin{array}{l}
\frac{1}{\lambda}\left(\frac{1}{2}\left(\sin \left(2 a_{m}\right) \cos (2 \Delta \theta)+\cos \left(2 a_{m}\right) \sin (2 \Delta \theta)\right) \cos ^{2}\left(\psi^{(0)}+\Delta \psi\right)\right) \\
-\frac{1}{\lambda_{R F}} \cos \left(a_{m}\right) \cos \left(\psi^{(0)}\right) \cdot\left(\sin \left(a_{m}+\Delta \theta\right) \cos \left(\psi^{(0)}+\Delta \psi\right)\right)
\end{array}\right)\right]=0
$$

Due to $\sum_{m=0}^{M-1} \sin \left(2 a_{m}\right)=0$ and $\sum_{m=0}^{M-1} \cos \left(2 a_{m}\right)=0$, which are proven in Appendix B, (10) is simplified to:

$$
\sum_{m=0}^{M-1}\left(\cos \left(a_{m}\right) \cos \left(\psi^{(0)}\right) \cdot\left(\sin \left(a_{m}+\Delta \theta\right) \cos \left(\psi^{(0)}+\Delta \psi\right)\right)\right)=0 .
$$

With angle addition and subtraction theorems, (11) is written as:

$$
\begin{aligned}
& \cos \left(\psi^{(0)}\right) \cos (\Delta \theta) \cos \left(\psi^{(0)}+\Delta \psi\right) \stackrel{\sum_{m=0}^{M-1}}{\cos \left(a_{m}\right) \sin \left(a_{m}\right)} \\
& +\cos \left(\psi^{(0)}\right) \sin (\Delta \theta) \cos \left(\psi^{(0)}+\Delta \psi\right) \sum_{m=0}^{M-1} \cos \left(a_{m}\right) \cdot \cos \left(a_{m}\right)=0 .
\end{aligned}
$$

From (A9) and (A12) in Appendix B, (12) can be rewritten as:

$$
\frac{M}{2} \cos \left(\psi^{(0)}\right) \sin (\Delta \theta) \cos \left(\psi^{(0)}+\Delta \psi\right)=0 .
$$

From (13), we get:

$$
\sin (\Delta \theta) \cos \left(\psi^{(0)}+\Delta \psi\right)=0
$$

From (14), the partial derivative of the cost function with respect to the azimuth is identically zero if either of the following conditions is true:

$$
\begin{gathered}
\sin (\Delta \theta)=0 \\
\cos \left(\psi^{(0)}+\Delta \psi\right)=0 .
\end{gathered}
$$

\subsection{Partial Derivative of the Cost Function with Respect to Elevation}

The partial derivative of the cost function with respect to $\psi$ is written as:

$$
\frac{\partial J(\theta, \psi)}{\partial \psi}=\sum_{m=0}^{M-1}\left[\begin{array}{c}
\sin \left(\frac{2 \pi r}{\lambda} \cos \left(\theta-\frac{2 \pi m}{M}\right) \cos (\psi)-\frac{2 \pi r}{\lambda_{R F}} \cos \left(\theta^{(0)}-\frac{2 \pi m}{M}\right) \cos \left(\psi^{(0)}\right)\right) \\
\cos \left(\theta-\frac{2 \pi m}{M}\right) \sin (\psi)
\end{array}\right] .
$$

The second necessary condition for $\theta=\theta^{(0)}+\Delta \theta, \psi=\psi^{(0)}+\Delta \psi$ to be a critical point of the cost function, $J(\theta, \psi)$, is that the partial derivative of the cost function, $J(\theta, \psi)$, in (2) with respect to the elevation, $\psi$, should be identically zero at $\theta=\theta^{(0)}+\Delta \theta, \psi=\psi^{(0)}+\Delta \psi$ :

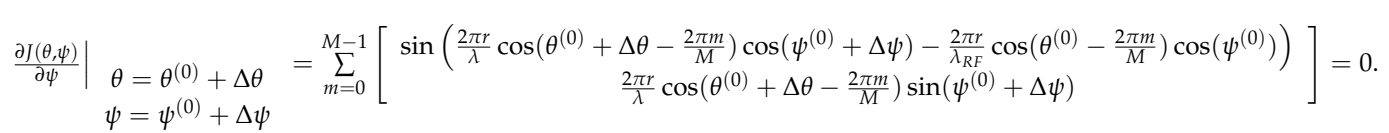

Due to $\sin (\phi) \approx \phi$ for $\phi \approx 0$, (18) can be written as:

$$
\sum_{m=0}^{M-1}\left[\begin{array}{c}
\left(\frac{2 \pi r}{\lambda} \cos \left(\theta^{(0)}+\Delta \theta-\frac{2 \pi m}{M}\right) \cos \left(\psi^{(0)}+\Delta \psi\right)-\frac{2 \pi r}{\lambda R F} \cos \left(\theta^{(0)}-\frac{2 \pi m}{M}\right) \cos \left(\psi^{(0)}\right)\right) \\
\frac{2 \pi r}{\lambda} \cos \left(\theta^{(0)}+\Delta \theta-\frac{2 \pi m}{M}\right) \sin \left(\psi^{(0)}+\Delta \psi\right)
\end{array}\right]=0 .
$$


Dividing both sides of (19) by $\frac{(2 \pi r)^{2}}{\lambda}$ yields:

$$
\sum_{m=0}^{M-1}\left[\begin{array}{c}
\left(\frac{1}{\lambda} \cos \left(a_{m}+\Delta \theta\right) \cos \left(\psi^{(0)}+\Delta \psi\right)-\frac{1}{\lambda_{R F}} \cos \left(a_{m}\right) \cos \left(\psi^{(0)}\right)\right) \\
\cdot \cos \left(a_{m}+\Delta \theta\right) \sin \left(\psi^{(0)}+\Delta \psi\right)
\end{array}\right]=0 .
$$

By the distributive law, (20) is written as:

$$
\sum_{m=0}^{M-1}\left(\begin{array}{l}
\frac{1}{\lambda} \cos \left(a_{m}+\Delta \theta\right) \cos \left(\psi^{(0)}+\Delta \psi\right) \cdot \cos \left(a_{m}+\Delta \theta\right) \sin \left(\psi^{(0)}+\Delta \psi\right) \\
-\frac{1}{\lambda_{R F}} \cos \left(a_{m}\right) \cos \left(\psi^{(0)}\right) \cdot \cos \left(a_{m}+\Delta \theta\right) \sin \left(\psi^{(0)}+\Delta \psi\right)
\end{array}\right)=0 .
$$

From the double angle formula for the sine function, (21) can be written as:

$$
\sin \left(\psi^{(0)}+\Delta \psi\right) \sum_{m=0}^{M-1}\left(\begin{array}{c}
\frac{1}{\lambda}\left(\frac{1+\cos \left(2 a_{m}+2 \Delta \theta\right)}{2}\right) \cos \left(\psi^{(0)}+\Delta \psi\right) \\
-\frac{1}{\lambda_{R F}} \cos \left(a_{m}\right) \cos \left(\psi^{(0)}\right) \cdot\left(\cos \left(a_{m}\right) \cos (\Delta \theta)-\sin \left(a_{m}\right) \sin (\Delta \theta)\right)
\end{array}\right)=0
$$

By Appendix B, due to $\sum_{m=0}^{M-1} \cos \left(2 a_{m}\right)=\sum_{m=0}^{M-1} \sin \left(2 a_{m}\right)=0$ and $\sum_{m=0}^{M-1} \sin \left(a_{m}\right) \cos \left(a_{m}\right)=0,(22)$ is simplified to:

$$
\sin \left(\psi^{(0)}+\Delta \psi\right) \frac{1}{\lambda} \frac{M}{2} \cos \left(\psi^{(0)}+\Delta \psi\right)-\sin \left(\psi^{(0)}+\Delta \psi\right) \frac{1}{\lambda_{R F}} \cos \left(\psi^{(0)}\right) \cos (\Delta \theta) \sum_{m=0}^{M-1} \cos ^{2}\left(a_{m}\right)=0
$$

Substituting (A8) in (23) yields:

$$
\sin \left(\psi^{(0)}+\Delta \psi\right) \frac{1}{\lambda} \frac{M}{2} \cos \left(\psi^{(0)}+\Delta \psi\right)-\sin \left(\psi^{(0)}+\Delta \psi\right) \frac{1}{\lambda_{R F}} \frac{M}{2} \cos \left(\psi^{(0)}\right) \cos (\Delta \theta)=0 .
$$

Since $\frac{M}{2}$ is not zero, by dividing both sides of (24) by $\frac{M}{2}$, we have:

$$
\sin \left(\psi^{(0)}+\Delta \psi\right)\left(\frac{1}{\lambda} \cos \left(\psi^{(0)}+\Delta \psi\right)-\frac{1}{\lambda_{R F}} \cos \left(\psi^{(0)}\right) \cos (\Delta \theta)\right)=0
$$

2.2.1. First Condition for the Partial Derivative with Respect to the Azimuth at $\theta=\theta^{(0)}+\Delta \theta, \psi=\psi^{(0)}+\Delta \psi$ to be Zero: $\sin (\Delta \theta)=0$

Note that, for (15) to be true, $\Delta \theta$ should be integer multiples of $\pi . \Delta \theta$ is an azimuth estimation error. It cannot be nonzero integer multiples of $\pi$ since the absolute value of the azimuth error should be much smaller than $\pi=180^{\circ}$. Therefore, from (15), we have:

$$
\Delta \theta=0 .
$$

Note that $\Delta \theta$ in (26) is independent of $\theta^{(0)}, \psi^{(0)}, \lambda$, and $\lambda_{R F}$, where $\theta^{(0)}$ and $\psi^{(0)}$ denote the azimuth and the elevation of an incident signal, respectively, and $\lambda_{R F}$ and $\lambda$ denote the wavelength of the incident signal and the nominal wavelength for the calculation of an array manifold. Therefore, the azimuth estimation error, $\Delta \theta$, is zero irrespective of in which direction the signal is incident and how different the frequency of the incident signal is from the nominal frequency used for the calculation of an array manifold.

From (26), the azimuth error is approximately equal to zero even when the array manifold frequency is different from the carrier frequency of the signal source in the absence of many uncertainties except the small frequency offset between the nominal frequency in the array manifold and the frequency of the incident signal. 
By substituting (26) in (25), we have:

$$
\sin \left(\psi^{(0)}+\Delta \psi\right)\left(\frac{1}{\lambda} \cos \left(\psi^{(0)}+\Delta \psi\right)-\frac{1}{\lambda_{R F}} \cos \left(\psi^{(0)}\right)\right)=0 .
$$

Applying angle addition and the subtraction theorem to (27) yields:

$$
\left(\sin \left(\psi^{(0)}\right) \cos (\Delta \psi)+\cos \left(\psi^{(0)}\right) \sin (\Delta \psi)\right)\left(\frac{1}{\lambda}\left(\cos \left(\psi^{(0)}\right) \cos (\Delta \psi)-\sin \left(\psi^{(0)}\right) \sin (\Delta \psi)\right)-\frac{1}{\lambda_{R F}} \cos \left(\psi^{(0)}\right)\right)=0 .
$$

Since (28) is not a polynomial equation in $\Delta \psi$, the Taylor approximation is used to make (28) a polynomial equation in $\Delta \psi$.

First, applying the approximations of $\cos (\Delta \psi) \approx 1, \sin (\Delta \psi) \approx \Delta \psi$ in (28) and a little manipulation yield:

$$
\left(\sin \left(\psi^{(0)}\right)+\cos \left(\psi^{(0)}\right) \Delta \psi\right)\left(\frac{1}{\lambda}\left(\cos \left(\psi^{(0)}\right)-\sin \left(\psi^{(0)}\right) \Delta \psi\right)-\frac{1}{\lambda_{R F}} \cos \left(\psi^{(0)}\right)\right)=0 .
$$

With the distributive law, (29) can be rewritten as:

$$
\begin{gathered}
(\Delta \psi)^{2}\left(\frac{1}{\lambda} \sin \left(\psi^{(0)}\right) \cos \left(\psi^{(0)}\right)\right) \\
+\Delta \psi\left(\frac{1}{\lambda} \cos ^{2}\left(\psi^{(0)}\right)-\frac{1}{\lambda} \sin ^{2}\left(\psi^{(0)}\right)-\frac{1}{\lambda_{R F}} \cos ^{2}\left(\psi^{(0)}\right)\right) \\
+\left(\frac{1}{\lambda}-\frac{1}{\lambda_{R F}}\right) \sin \left(\psi^{(0)}\right) \cos \left(\psi^{(0)}\right)=0 .
\end{gathered}
$$

If $\psi^{(0)}$ is equal to zero or $\frac{\pi}{2}$, the coefficient of the second order term, $\sin \left(\psi^{(0)}\right) \cos \left(\psi^{(0)}\right)$, and the constant term, $\left(\frac{1}{\lambda}-\frac{1}{\lambda_{R F}}\right) \sin \left(\psi^{(0)}\right) \cos \left(\psi^{(0)}\right)$, are equal to zero. In that case, (30) is no longer a second order equation.

For $\psi^{(0)}=0^{\circ},(30)$ reduces to:

$$
\left(\frac{1}{\lambda}-\frac{1}{\lambda_{R F}}\right) \Delta \psi=0
$$

$\Delta \psi$ should be zero unless $\lambda$ is equal to $\lambda_{R F}$.

For $\psi^{(0)}=90^{\circ},(30)$ reduces to:

$$
\frac{1}{\lambda} \Delta \psi=0
$$

from which $\Delta \psi$ should be zero. If $\psi^{(0)}$ is not equal to zero or $\frac{\pi}{2},(30)$ is a second order equation, and the roots of (30) are obtained from the quadratic formula.

From (30), the constant term of the quadratic equation is given by:

$$
\left(\frac{1}{\lambda}-\frac{1}{\lambda_{R F}}\right) \sin \left(\psi^{(0)}\right) \cos \left(\psi^{(0)}\right)
$$

If the wavelength associated with the frequency of the incident signal, $\lambda_{R F}$, is equal to the wavelength associated with the nominal frequency for the calculation of the array manifold, $\lambda,(31)$ reduces to identically zero. In this case, (30) is simplified to:

$$
\begin{aligned}
& (\Delta \psi)^{2}\left(\frac{1}{\lambda} \sin \left(\psi^{(0)}\right) \cos \left(\psi^{(0)}\right)\right)+\Delta \psi\left(\frac{1}{\lambda} \cos ^{2}\left(\psi^{(0)}\right)-\frac{1}{\lambda} \sin ^{2}\left(\psi^{(0)}\right)-\frac{1}{\lambda_{R F}} \cos ^{2}\left(\psi^{(0)}\right)\right)=0 \\
& \Delta \psi\left(\Delta \psi\left(\frac{1}{\lambda} \sin \left(\psi^{(0)}\right) \cos \left(\psi^{(0)}\right)\right)+\left(\frac{1}{\lambda} \cos ^{2}\left(\psi^{(0)}\right)-\frac{1}{\lambda} \sin ^{2}\left(\psi^{(0)}\right)-\frac{1}{\lambda_{R F}} \cos ^{2}\left(\psi^{(0)}\right)\right)\right)=0 .
\end{aligned}
$$

For $\lambda_{R F}=\lambda$, from (33), it can be seen that $\Delta \psi=0$ is always a solution of (30). Since $\Delta \theta$ is zero from (26), both $\Delta \theta$ and $\Delta \psi$ are identically zero for $\lambda_{R F}=\lambda$, which is consistent with the following 
intuition: for the given true azimuth of $\theta^{(0)}$ and the true elevation of $\psi^{(0)}$ in (2), we are concerned with what values of $\theta$ and $\psi$ maximize the cost function in (2). Intuitively, it can be seen that, for $\lambda_{R F}=\lambda$, the argument of the cosine function in (2) is zero if $\theta$ and $\psi$ are given by $\theta^{(0)}$ and $\psi^{(0)}$, respectively. Since the cosine function is maximized when the argument is zero, for $\lambda_{R F}=\lambda$, the cost function in (2) is maximized when $\theta$ and $\psi$ are given by $\theta^{(0)}$ and $\psi^{(0)}$, respectively. $\theta=\theta^{(0)}$ and $\psi=\psi^{(0)}$ are equivalent to $\Delta \theta=0$ and $\Delta \psi=0$.

Since (30) is a quadratic equation in $\Delta \psi$, the roots can be easily obtained from the quadratic formula. Explicit expressions of two roots of (30) are given by:

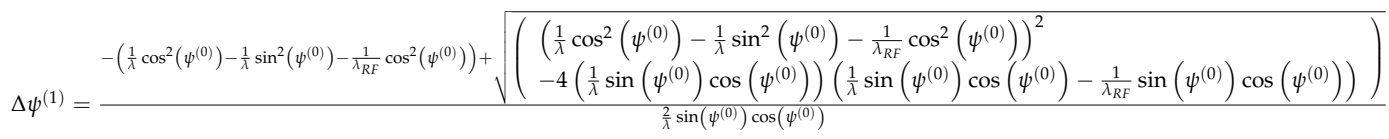

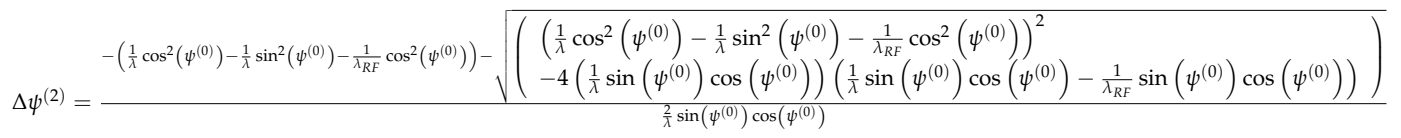

To choose one root out of the two roots in (34), the cost function in (2) is evaluated with $\theta=$ $\theta^{(0)}+\Delta \theta, \psi=\psi^{(0)}+\Delta \psi$. Note that $\theta$ should be equal to $\theta^{(0)}$ due to $\Delta \theta=0$ in (26). Out of two roots in (34), the root resulting in a greater cost function in (2) is finally selected: if $J\left(\theta^{(0)}, \psi^{(0)}+\Delta \psi^{(1)}\right)$ is greater than $J\left(\theta^{(0)}, \psi^{(0)}+\Delta \psi^{(2)}\right), \Delta \psi^{(1)}$ is chosen as $\Delta \psi$. If $J\left(\theta^{(0)}, \psi^{(0)}+\Delta \psi^{(1)}\right)$ is smaller than $J\left(\theta^{(0)}, \psi^{(0)}+\Delta \psi^{(2)}\right), \Delta \psi^{(2)}$ is chosen as $\Delta \psi$.

In summary, the expressions for the estimation errors of $\Delta \theta$ and $\Delta \psi$, under the approximations of $\sin (\Delta \psi) \approx \Delta \psi$ and $\cos (\Delta \psi) \approx 1$, are given by (26) and (34). While the approximations of $\sin (\Delta \psi) \approx \Delta \psi$ and $\cos (\Delta \psi) \approx 1$ are employed to get (34), no approximation is used in getting (26)

Note that $\Delta \psi$ in (34) is dependent on $\psi^{(0)}, \lambda$ and $\lambda_{R F}$, not on $\theta^{(0)}$ : under the approximations of $\sin (\Delta \psi) \approx \Delta \psi$ and $\cos (\Delta \psi) \approx 1$, the elevation estimation error, $\Delta \psi$, is dependent on how different the frequency of the incident signal is from the nominal frequency used for the calculation of an array manifold, and the elevation error is also dependent on the true elevation of the incident signal, $\psi^{(0)}$, not the true azimuth of the incident signal, $\theta^{(0)}$. Therefore, for given $\lambda$ and $\lambda_{R F}$, the elevation estimation error, $\Delta \psi$, is only dependent on the true elevation of the incident signal, $\psi^{(0)}$, not the true azimuth of the incident signal, $\theta^{(0)}$.

Although $\cos (\Delta \psi) \approx 1$ is quite accurate when $\Delta \psi$ is close to zero, it becomes inaccurate with the increase of the absolute value of $\Delta \psi$. To circumvent this problem, higher order approximation for the cosine function can be adopted.

Substituting approximations of $\cos (\Delta \psi) \approx 1-\frac{\Delta \psi^{2}}{2}$ and $\sin (\Delta \psi) \approx \Delta \psi$ in (25) and rearranging terms yield:

$$
\begin{gathered}
(\Delta \psi)^{4}\left[\frac{1}{4} \frac{1}{\lambda} \cos \left(\psi^{(0)}\right) \sin \left(\psi^{(0)}\right)\right] \\
+(\Delta \psi)^{3}\left[-\frac{1}{2 \lambda} \cos ^{2}\left(\psi^{(0)}\right)+\frac{1}{2 \lambda} \sin ^{2}\left(\psi^{(0)}\right)\right] \\
+(\Delta \psi)^{2}\left[\begin{array}{l}
-\frac{1}{\lambda} \cos \left(\psi^{(0)}\right) \sin \left(\psi^{(0)}\right)-\frac{1}{2} \frac{1}{\lambda} \cos \left(\psi^{(0)}\right) \sin \left(\psi^{(0)}\right) \\
-\frac{1}{\lambda} \sin \left(\psi^{(0)}\right) \cos \left(\psi^{(0)}\right)+\frac{1}{\lambda_{R F}} \sin \left(\psi^{(0)}\right) \cos \left(\psi^{(0)}\right)
\end{array}\right] \\
+\Delta \psi\left[\frac{1}{\lambda} \cos ^{2}\left(\psi^{(0)}\right)-\frac{1}{\lambda} \sin ^{2}\left(\psi^{(0)}\right)-\frac{1}{\lambda_{R F}} \cos ^{2}\left(\psi^{(0)}\right)\right] \\
+\frac{1}{\lambda} \cos \left(\psi^{(0)}\right) \sin \left(\psi^{(0)}\right)-\frac{1}{\lambda_{R F}} \cos \left(\psi^{(0)}\right) \sin \left(\psi^{(0)}\right)=0 .
\end{gathered}
$$

For $\lambda_{R F}=\lambda$, since the constant term of the fourth order equation in (35) reduces to zero, (35) is written as: 


$$
\begin{aligned}
& (\Delta \psi)^{4}\left[\frac{1}{4} \frac{1}{\lambda} \cos \left(\psi^{(0)}\right) \sin \left(\psi^{(0)}\right)\right]+(\Delta \psi)^{3}\left[-\frac{1}{2 \lambda} \cos ^{2}\left(\psi^{(0)}\right)+\frac{1}{2 \lambda} \sin ^{2}\left(\psi^{(0)}\right)\right] \\
& +(\Delta \psi)^{2}\left[-\frac{1}{2} \frac{1}{\lambda} \cos \left(\psi^{(0)}\right) \sin \left(\psi^{(0)}\right)-\frac{1}{\lambda} \sin \left(\psi^{(0)}\right) \cos \left(\psi^{(0)}\right)\right]+\Delta \psi\left[-\frac{1}{\lambda} \sin ^{2}\left(\psi^{(0)}\right)\right]=0 .
\end{aligned}
$$

It is clear that $\Delta \psi=0$ is always one of the solutions of (36) under the approximation of $\cos (\Delta \psi) \approx 1-\frac{\Delta \psi^{2}}{2}, \sin (\Delta \psi) \approx \Delta \psi$, as well as under the approximation of $\cos (\Delta \psi) \approx 1, \sin (\Delta \psi)=\Delta \psi$. Consequently, following the same reasoning used to get the elevation estimation error, $\Delta \psi$, under the approximations of $\cos (\Delta \psi) \approx 1, \sin (\Delta \psi)=\Delta \psi$, it is true that $\Delta \theta$ and $\Delta \psi$ for $\lambda_{R F}=\lambda$ are given by $\Delta \theta=0$ and $\Delta \psi=0$ : without frequency offset, the elevation error, $\Delta \psi$, as well as the azimuth estimation error, $\Delta \theta$, are identically zero.

(35) is a fourth order equation in $\Delta \psi$. All the real roots of (35) are obtained, and each real root, $\Delta \psi$, is used for evaluating the cost function in (2) with $\theta=\theta^{(0)}+\Delta \theta, \psi=\psi^{(0)}+\Delta \psi \cdot \theta=\theta^{(0)}$ should be used since (26) should be true for the partial derivative of the cost function with respect to the azimuth to be zero. Out of the real roots of the fourth order Equation (35), the root maximizing the cost function value is selected as the elevation error, $\Delta \psi$.

In summary, the expressions for the estimation errors of $\Delta \theta$ and $\Delta \psi$, under the approximations of $\sin (\Delta \psi) \approx \Delta \psi$ and $\cos (\Delta \psi) \approx 1-\frac{\Delta \psi^{2}}{2}$, are given by (26) and a root out of the four roots of (35)

From (35), the coefficients of the fourth order equation in $\Delta \psi$ are dependent on $\psi^{(0)}, \lambda$, and $\lambda_{R F}$, not on $\theta^{(0)}$, which is also true under the approximations of $\sin (\Delta \psi) \approx \Delta \psi$ and $\cos (\Delta \psi) \approx 1$. Under the approximations of $\sin (\Delta \psi) \approx \Delta \psi$ and $\cos (\Delta \psi) \approx 1-\frac{\Delta \psi^{2}}{2}$, the elevation estimation error, $\Delta \psi$, is dependent on how different the wavelength of the incident signal, $\lambda_{R F}$, is from the nominal wavelength used for the calculation of the array manifold, $\lambda$. The elevation error, $\Delta \psi$, is also dependent on the true elevation of the incident signal, $\psi^{(0)}$, not the true azimuth of the incident signal, $\theta^{(0)}$. Consequently, from (26) and (34), the dependence of the elevation estimation error on the true elevation, the wavelength of the incident signal, and the nominal wavelength for the array manifold is the same both for the approximations of $\sin (\Delta \psi) \approx \Delta \psi, \cos (\Delta \psi) \approx 1$ and for the approximations of $\sin (\Delta \psi) \approx \Delta \psi, \cos (\Delta \psi) \approx 1-\frac{\Delta \psi^{2}}{2}$.

How accurate the first order Taylor approximation and the second order approximation are highly depends on how large the elevation estimation error, $\Delta \psi$, is: the accuracy of the first order approximation, $\cos (\Delta \psi) \approx 1$, degrades as $\cos (\Delta \psi) \approx 1$ increases.

For improvement of the accuracy of the Taylor approximation, the second order approximation, $\cos (\Delta \psi) \approx 1-\frac{\Delta \psi^{2}}{2}$, can be applied. Note that the difference between the first order Taylor approximation and the second order Taylor approximation is $\frac{\Delta \psi^{2}}{2}$, implying that the superiority of the second order approximation over the first order approximation is dominant for large $\Delta \psi$ :

For small elevation estimation error, adopting only the first order Taylor approximation results in the agreement between the simulation-based results and the analytically-derived results. Inconsistency between the simulation-based results and the analytically-derived results increases with the increase of the absolute value of $\Delta \psi$, implying that the second order Taylor approximation should be adopted for the agreement of the simulation-based results and the analytically-derived results when the absolute value of elevation estimation error, $\Delta \psi$, is large.

For small $\Delta \psi$, since $\frac{\Delta \psi^{2}}{2}$ is small, the superiority of the second order approximation over the first order approximation is very small, implying that the second order approximation need not be adopted for small elevation estimation error.

2.2.2. Second Condition for the Partial Derivative with Respect to the Azimuth at $\theta=\theta^{(0)}+\Delta \theta, \psi=\psi^{(0)}+\Delta \psi$ to be Zero: $\operatorname{Cos}\left(\psi^{(0)}+\Delta \psi\right)=0$

From (16), we have: 


$$
\sin \left(\psi^{(0)}+\Delta \psi\right)=1
$$

Substituting (16) and (37) in (25) yields:

$$
\cos \left(\psi^{(0)}\right) \cos (\Delta \theta)=0 .
$$

For (38) to be true, $\cos (\Delta \theta)$ should be equal to zero, implying that $\Delta \theta$ should be odd multiples of $\frac{\pi}{2}$. As previously stated, $\Delta \theta$ is much smaller than $\frac{\pi}{2}$ since $\Delta \theta$ denotes a small azimuth estimation error. Therefore, (38) cannot be true, which implies that, for $\cos \left(\psi^{(0)}+\Delta \psi\right)=0$ in (16), it is impossible to find $\Delta \theta$ satisfying (25), which is the constraint that the partial derivative of the cost function with respect to elevation should be zero.

\section{Summary of the Analytic Derivation of the Azimuth Estimation Error and the Elevation Estimation Error}

Necessary conditions for minimizing the cost function are that both the partial derivative of the cost function with respect to $\theta$ and that with respect to $\psi$ should simultaneously be zero. Based on these two constraints, expressions of $\Delta \theta$ and $\Delta \psi$ are derived under the Taylor approximation.

It turns out that the partial derivative of the cost function of an interferometer algorithm with respect to $\theta$ at $\theta=\theta^{(0)}+\Delta \theta, \psi=\psi^{(0)}+\Delta \psi$ is zero if either of two condition is satisfied:

$$
\begin{gathered}
\Delta \theta=0 \\
\cos \left(\psi^{(0)}+\Delta \psi\right)=0 .
\end{gathered}
$$

\subsection{First Condition: $\Delta \theta=0$}

In the first case of $\Delta \theta=0, \Delta \theta=0$ implies that there should be no estimation error in the azimuth. On the other hand, $\Delta \theta=0$ does not impose any restriction on the elevation estimation error, which implies that, under the first order approximation, the partial derivative of the cost function of an interferometer algorithm with respect to $\theta$ is zero for $\Delta \theta=0$, irrespective of $\Delta \psi$, even in the presence of frequency offset between the frequency of the incident signal and the nominal frequency adopted in calculating the array manifold. As previously stated, since the requirement that the partial derivative of the cost function with respect to $\theta$ should be zero at $\theta=\theta^{(0)}+\Delta \theta, \psi=\psi^{(0)}+\Delta \psi$ does not impose any constraint on the elevation error, $\Delta \psi$, the constraint on the elevation error, $\Delta \psi$, is imposed from the fact that the partial derivative of the cost function with respect to $\psi$ should also be zero. Substituting $\Delta \theta=0$ in the equation resulting from the constraint that the partial derivative of the cost function with respect to $\psi$ should be zero, this constraint reduces to a quadratic equation or a fourth order equation in $\Delta \psi$, depending on the approximations employed:

$$
\begin{array}{ll}
\text { Second order equation in } \Delta \psi & \cos (\Delta \psi) \approx 1, \sin (\Delta \psi) \approx \Delta \psi \\
\text { Fourth order equation in } \Delta \psi & \cos (\Delta \psi) \approx 1-\frac{\Delta \psi^{2}}{2}, \sin (\Delta \psi) \approx \Delta \psi
\end{array}
$$

Out of two roots of the second order equation or out of four roots of the fourth order equation, $\Delta \psi$ maximizing the cost function is selected. Therefore, $\Delta \psi$ can easily be obtained from a quadratic formula.

\subsection{Second Condition: $\cos \left(\psi^{(0)}+\Delta \psi\right)=0$}

Substituting $\cos \left(\psi^{(0)}+\Delta \psi\right)=0$ in the constraint that the partial derivative of cost function of an interferometer algorithm with respect to $\psi$ at $\theta=\theta^{(0)}+\Delta \theta, \psi=\psi^{(0)}+\Delta \psi$ should be zero, we get the following equation in $\Delta \theta$ :

$$
\cos \left(\psi^{(0)}\right) \cos (\Delta \theta)=0
$$

It turns out that no $\Delta \theta$ satisfies (41) since $|\Delta \theta|$ is much less than $\frac{\pi}{2}$. 


\section{Simulation Results}

In this section, simulation-based estimation errors are compared with analytically-derived estimation errors to validate the derived expressions for the analytically-derived estimation errors. The simulation condition is tabulated in Table 1.

The results for the true azimuth and true elevation of $\left(\theta^{(0)}=120^{\circ}, \psi^{(0)}=0^{\circ}\right),\left(\theta^{(0)}=120^{\circ}, \psi^{(0)}=\right.$ $\left.20^{\circ}\right),\left(\theta^{(0)}=120^{\circ}, \psi^{(0)}=50^{\circ}\right)$, and $\left(\theta^{(0)}=120^{\circ}, \psi^{(0)}=80^{\circ}\right)$ are illustrated in Figures $2 \mathrm{a}, \mathrm{b}$ and $3 \mathrm{a}, \mathrm{b}$, respectively.

Remember that, from (26), the analytic azimuth estimation error is always zero. The elevation estimation error with "Analytic error (first order approx)" and "Analytic error (second order approx)" are obtained from (34) and (35), respectively.

For the elevation estimation error with "Simulation error", the exhaustive evaluation of (2), for given $\theta^{(0)}$ and $\psi^{(0)}$, with respect to two variables of $\theta$ and $\psi$ is performed, and $\theta$ and $\psi$ maximizing the cost function in (2) are chosen to be the estimate of the azimuth and the estimate of the elevation, respectively.

The difference between the estimate of the azimuth and the true azimuth is defined as the simulation-based azimuth estimation error. The simulation-based elevation estimation error is similarly defined. In Figure 2, the $y$-axis represents the azimuth and elevation error and the $x$-axis represents the carrier frequency of the signal source. Note that the frequency of the array manifold is equal to $1 \mathrm{GHz}$, whose wavelength is denoted by $\lambda$

Table 1. Simulation conditions.

\begin{tabular}{cc}
\hline Parameter & Value \\
\hline Number of sensors & 5 \\
Array architecture & UCA \\
Azimuth and elevation search step & $0.25^{\circ}$ \\
\hline
\end{tabular}
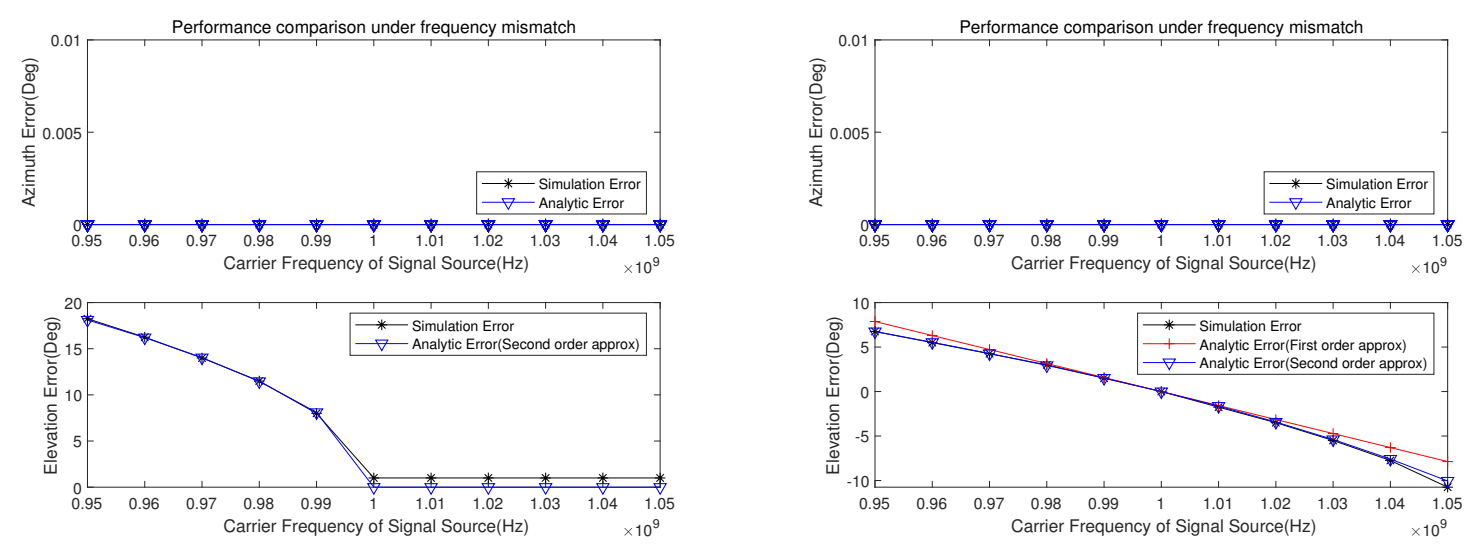

(a) $\theta^{(0)}=120^{\circ}, \quad \psi^{(0)}=0^{\circ}$

(b) $\theta^{(0)}=120^{\circ}, \quad \psi^{(0)}=20^{\circ}$

Figure 2. Analytic and simulated azimuth and elevation error with respect to the carrier frequency of the signal source for $\psi^{(0)}=0^{\circ}$ and $\psi^{(0)}=20^{\circ}$.

Under the approximations of $\cos (\Delta \psi) \approx 1, \sin (\Delta) \approx \Delta \psi$, from (3), $\Delta \psi$ for $\psi^{(0)}=0^{\circ}$ is zero, which is why the analytic elevation error with the legend "Analytic error (first order approx)" is not shown in the lower plot of Figure 2a. Remember that $\Delta \psi$ denotes analytic elevation error.

The results with the legend "Analytic error (second order approx)" show an excellent agreement with the simulation-based results in Figure 2a, which validates our derivation. 
As shown in the lower plot of Figure 2b, discrepancy between analytic results with the legend "Analytic error (first order approx)" in Figure $2 \mathrm{~b}$ and the simulation error with the legend "Simulation error" occurs for an incident signal of $\theta^{(0)}=120^{\circ}, \psi^{(0)}=20^{\circ}$, and the discrepancy increases with the increase of $|\Delta \psi|$, which is the absolute value of the simulation-based elevation estimation error.

The simulation-based error is obtained from the cost function of an interferometer algorithm without any approximation. On the other hand, to get the analytic error, the approximations of $\cos (\Delta \psi) \approx 1, \sin (\Delta \psi) \approx \Delta \psi$ are applied. Therefore, for the discrepancy between the simulated error and the analytically-derived error to be small, the approximation error in the Taylor series expansion should be small. In the approximation of $\cos (\Delta \psi) \approx 1$, the absolute value of the error is bounded by $\frac{\Delta \psi^{2}}{2}$, implying that the approximation error is not small enough to be negligible for large $\Delta \psi$.

As shown in the results with the legend "Simulation error" in the lower plot of Figure $2 b$, the largest value of the absolute value of $\Delta \psi$, for the small true elevation angle of $\psi^{(0)}=20^{\circ}$, is approximately $10^{\circ}$ for the carrier frequency of $1.05 \mathrm{GHz}$. Considering the worst case of $\Delta \psi=10^{\circ}$, the approximation $\cos (\Delta \psi) \approx 1$ induces an error, which can be expressed as the absolute value of $1-\cos \left(10^{\circ}\right)$. Since, in deriving the results with the legend "Analytic error (first order approx)", $\cos (\Delta \psi) \approx 1$ is used, the approximation of $\cos (\Delta \psi) \approx 1$ induces an error of the absolute value of $1-\cos \left(10^{\circ}\right)$ for $\Delta \psi=10^{\circ}$, which is why the largest discrepancy between the results with the legend "Simulation error" and the results with the legend "Analytic error (first order approx)" occurs at the carrier frequency of $1.05 \mathrm{GHz}$. The discrepancy between the results with the legend "Simulation error" and the results with the legend "Analytic error (first order approx)" at the other carrier frequencies is smaller than the discrepancy at the carrier frequency of $1.05 \mathrm{GHz}$ : from the simulation-based results in the lower plot of Figure $2 b$, the absolute value of the elevation estimation error, $\Delta \psi$, is greatest at the carrier frequency of $1.05 \mathrm{GHz}$, implying that the largest discrepancy should occur at that frequency. Remember that, in the approximation of $\cos (\Delta \psi) \approx 1$, the approximation error increases with the increase of the absolute value of $\Delta \psi$.

To reduce the approximation error in $\cos (\Delta \psi) \approx 1$ for large $\Delta \psi, \cos (\Delta \psi) \approx 1-\frac{\Delta \psi^{2}}{2}$ is adopted, and the associated results with the legend "Analytic error (second order approx)" are illustrated in the lower plot of Figure $2 b$.

Since the approximation error in $\cos (\Delta \psi) \approx 1-\frac{\Delta \psi^{2}}{2}$ is much smaller than that in $\cos (\Delta \psi) \approx 1$ for large $\Delta \psi$, the agreement between the results with the legend "Analytic error (second order approx)" and the simulated results is much better than the agreement between the results with the legend "Analytic error (first order approx)" and the simulated results. This observation is consistent with the fact that the approximation $\cos (\Delta \psi) \approx 1-\frac{\Delta \psi^{2}}{2}$ is quite accurate for the worst case of $\Delta \psi=10^{\circ}$ in comparison with the approximation $\cos (\Delta \psi) \approx 1$. Note that the superiority of the approximation $\cos (\Delta \psi) \approx 1-\frac{\Delta \psi^{2}}{2}$ over the approximation $\cos (\Delta \psi) \approx 1$ is clear when the absolute value of the elevation estimation error, $\Delta \psi$, is large.

The agreement between the analytically-derived results and the simulation-based results in the lower plot of Figure $2 \mathrm{~b}$ can be improved by reducing the approximation error. This can be achieved by adopting the second order Taylor series, not the first order Taylor series expansion, in getting the analytic error. In the second order approximation of $\cos (\Delta \psi) \approx 1-\frac{\Delta \psi^{2}}{2}$, the absolute value of the error is bounded by $\frac{\Delta \psi^{4}}{4}$.

In the lower plot of Figure $2 b$, the results for the incident signal of azimuth $=120^{\circ}$, elevation $=10^{\circ}$ are illustrated, where the analytic error with the legend "Analytic error (second order approx)" is obtained from the second order Taylor approximation, not the first order Taylor approximation. The analytic results with the legend "Analytic error (second order approx)" are closer to the simulation-based results than the analytic results with the legend "Analytic error (first order approx)", illustrating the improvement by adopting $\cos (\Delta \psi) \approx 1-\frac{\Delta \psi^{2}}{2}$, rather than $\cos (\Delta \psi) \approx 1$. 
It is clear that the agreement between the analytic results with the legend "Analytic error (second order approx)" and simulation-based results is better than the agreement between the analytic results with the legend "Analytic error (First order approx)" in the lower plots of Figures $2 b$ and $3 a, b$.

In the results with "Simulation error" in the lower plots of Figures 3a,b., the largest value of the absolute value of the elevation estimation error, $\Delta \psi$, is approximately $2^{\circ}$ and $0.5^{\circ}$, respectively, both of which are much smaller than $10^{\circ}$. Remember that the largest value of the absolute value of the simulation-based elevation estimation error in Figure $2 \mathrm{~b}$ is approximately $10^{\circ}$.

The error bound of the absolute value of the error in the approximation $\cos (\Delta \psi) \approx 1$ is given by $\frac{\Delta \psi^{2}}{2}$, which gets smaller with the decrease of $|\Delta \psi|$. Therefore, the approximation error due to the first order Taylor expansion in getting the analytic error gets smaller with the decrease of $|\Delta \psi|$. The discrepancies between the simulated error and the analytically-derived error in the lower plot of Figure $3 \mathrm{a}$ is smaller than that in the lower plot of Figure $2 \mathrm{~b}$ since simulation-based $|\Delta \psi|$ in the lower plot of Figure 3a is smaller than that in the lower plot of Figure 2b, both for the results with "Analytic error (first order approx)" and for the results with "Analytic error (second order approx)". Similarly, both for the results with "Analytic error (first order approx)" and for the results with "Analytic error (second order approx)", the discrepancy between the simulated error and the analytically-derived error in the lower plot of Figure $3 b$ is smaller than that in the lower plot of Figure 3a since simulation-based $|\Delta \psi|$ in the lower plot of Figure $3 \mathrm{~b}$ is smaller than that in the lower plot of Figure 3a.
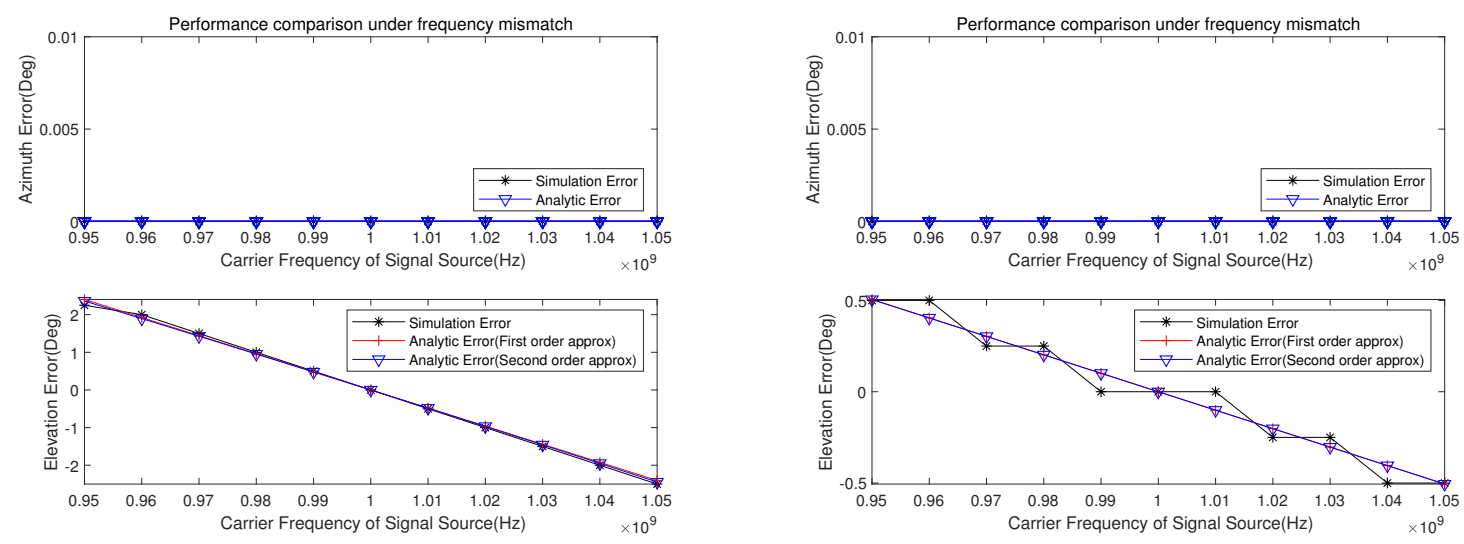

(a) $\theta^{(0)}=120^{\circ}, \quad \psi^{(0)}=50^{\circ}$

(b) $\theta^{(0)}=120^{\circ}, \quad \psi^{(0)}=80^{\circ}$

Figure 3. Analytic and simulated azimuth and elevation error with respect to the carrier frequency of the signal source for $\psi^{(0)}=50^{\circ}$ and $\psi^{(0)}=80^{\circ}$.

In Figures 4 and 5, the $x$-axis, the $y$-axis, and the $z$-axis represent the true azimuth angle of the incident signal, the true elevation angle of the incident signal, and the estimation error, respectively. The true azimuth angle of the incident signal increases from zero to 359 degrees in increments of $5^{\circ}$. The true elevation angle of the incident signal increases from zero to 89 degrees in increments of $5^{\circ}$. The frequency of the array manifold is $1 \mathrm{GHz}$, and the carrier frequency of the incident signal is $950 \mathrm{MHz}$. Figure 4 shows the simulation-based azimuth estimation error with respect to the true azimuth angle and the true elevation angle of the incident signal.

Figure 5a illustrates the simulation-based elevation estimation error with respect to the true azimuth angle and the true elevation angle of the incident signal. Figure $5 \mathrm{~b}$ shows the analytically-derived elevation error. It is shown that the error of the azimuth is always zero regardless of the true value of the azimuth and the elevation, and the error of the elevation depends only on the true elevation angle of an incident signal not on the true azimuth of an incident signal. 


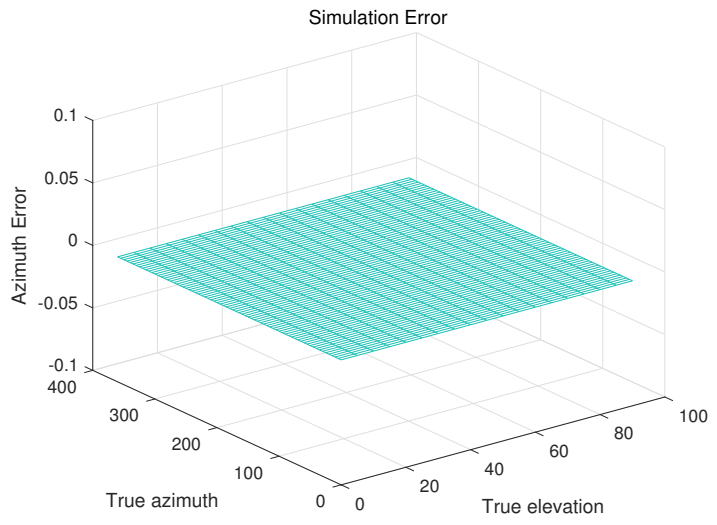

(a)

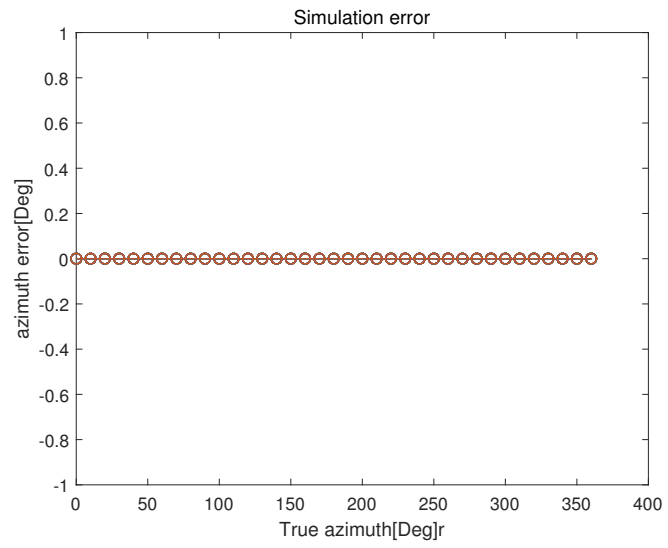

(b)

Figure 4. Simulation-based azimuth error $\left(f_{R F}=\frac{c}{\lambda_{R F}}=950 \mathrm{MHz}\right)$. (a) Simulation error elevation with respect to the true azimuth and true elevation. (b) Simulation error with respect to the true azimuth.

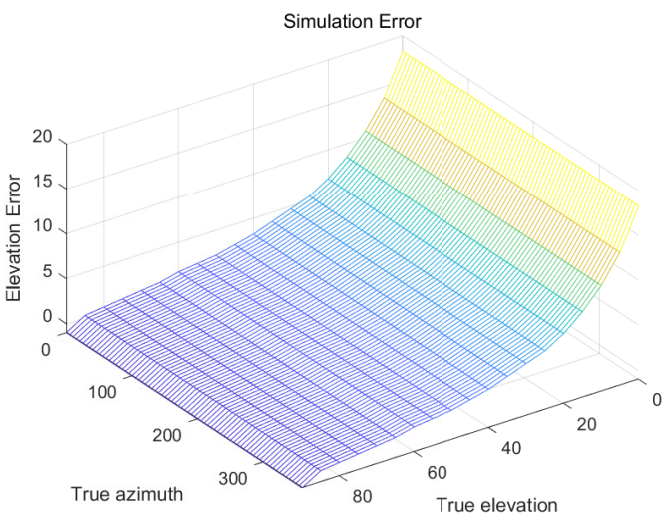

(a) Simulation error

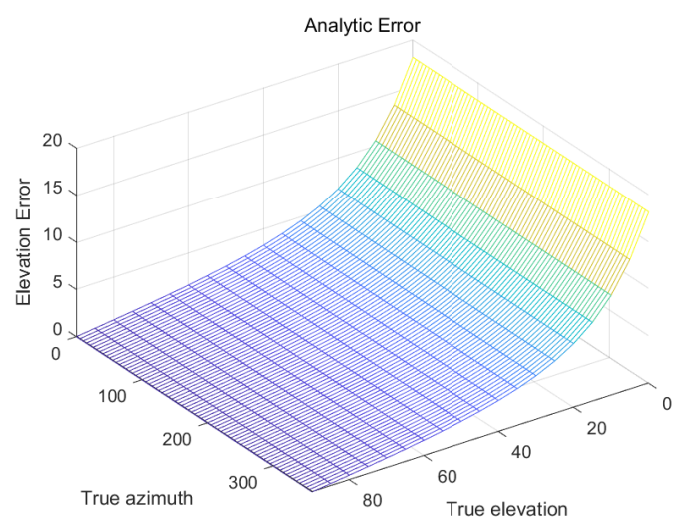

(b) Analytic error

Figure 5. Analytic elevation error and simulation-based elevation error with respect to the true azimuth and the elevation of the incident signal $\left(f_{R F}=\frac{c}{\lambda_{R F}}=950 \mathrm{MHz}\right)$.

Figures 6-10 show the simulation-based elevation error and analytical elevation error with respect to the true elevation. In Figure $6 \mathrm{a}, \mathrm{b}$, the frequencies of the incident signal are $950 \mathrm{MHz}$ and $960 \mathrm{MHz}$, respectively. Figures 7a,b, 8a,b, 9a,b, and 10a,b illustrate the results for $970 \mathrm{MHz}$, $980 \mathrm{MHz}, 990 \mathrm{MHz}, 1010 \mathrm{MHz}, 1020 \mathrm{MHz}, 1030 \mathrm{MHz}, 1040 \mathrm{MHz}$, and $1050 \mathrm{MHz}$, respectively. The analytically-derived elevation estimation errors show excellent agreement with the simulation-based elevation estimation error in Figures 6-10. For the evaluation of the analytically-derived elevation estimation error, the approximations of $\cos (\Delta \psi) \approx 1-\frac{\Delta \psi^{2}}{2}$ and $\sin (\Delta \psi) \approx \Delta \psi$ are adopted. 


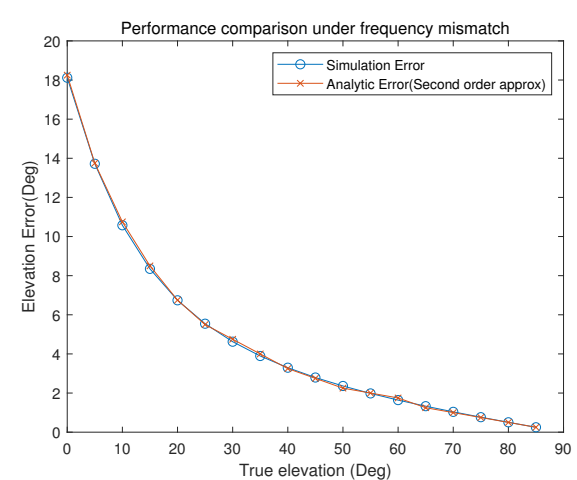

(a) RF: $950 \mathrm{MHz}$

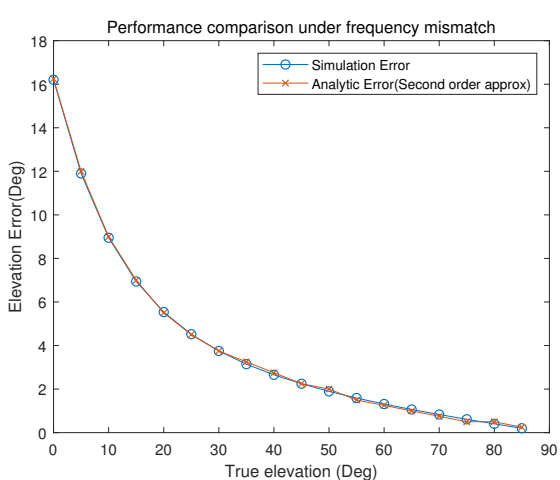

(b) RF: $960 \mathrm{MHz}$

Figure 6. Analytically-derived elevation error and simulation-based error with respect to the true elevation of the incident signal $\left(f_{R F}=\frac{c}{\lambda_{R F}}=950 \mathrm{MHz}\right.$ and $\left.960 \mathrm{MHz}\right)$.

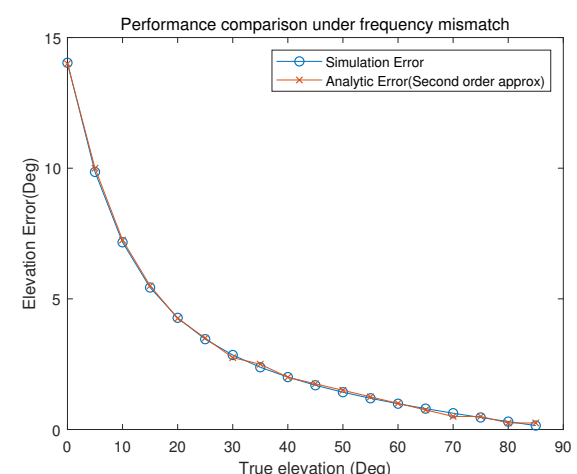

(a) RF: $970 \mathrm{MHz}$

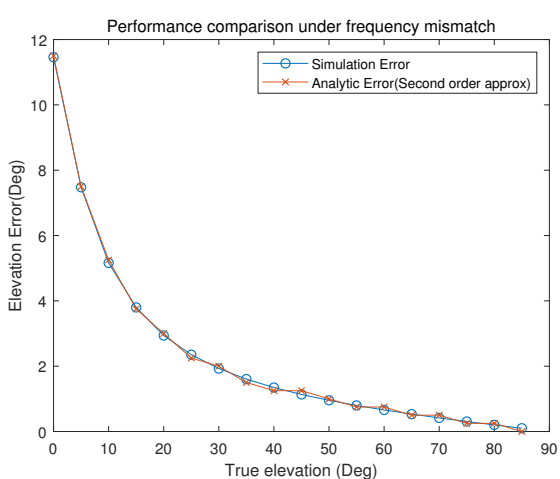

(b) RF: $980 \mathrm{MHz}$

Figure 7. Analytically-derived elevation error and simulation-based error with respect to the true elevation of the incident signal $\left(f_{R F}=\frac{c}{\lambda_{R F}}=970 \mathrm{MHz}\right.$ and $\left.980 \mathrm{MHz}\right)$.

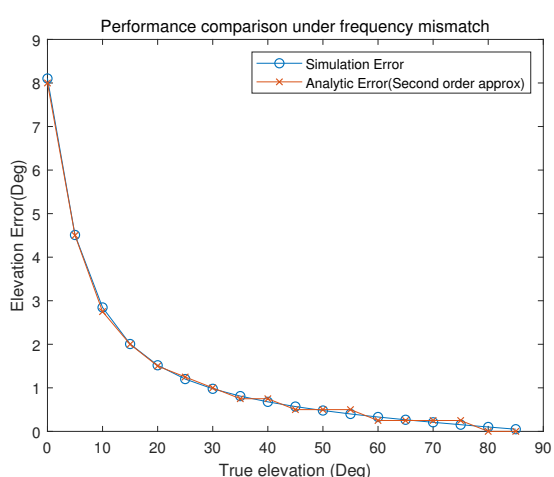

(a) RF: $990 \mathrm{MHz}$

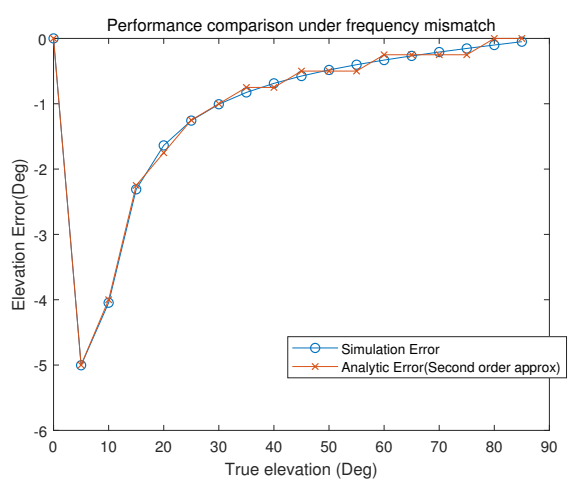

(b) RF: $1010 \mathrm{MHz}$

Figure 8. Analytically-derived elevation error and simulation-based error with respect to the true elevation of the incident signal $\left(f_{R F}=\frac{c}{\lambda_{R F}}=990 \mathrm{MHz}\right.$ and $\left.1010 \mathrm{MHz}\right)$. 


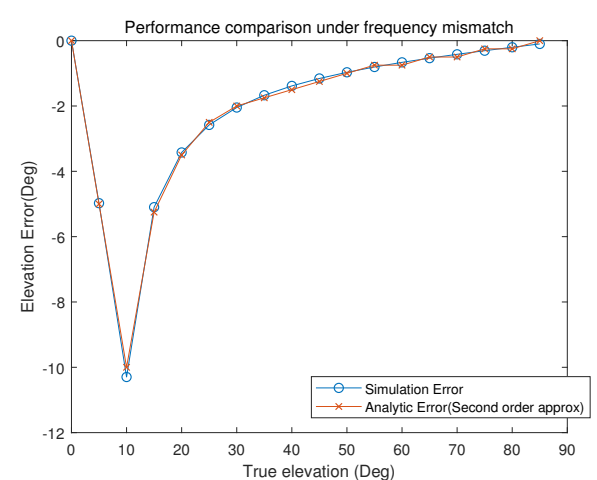

(a) RF: $1020 \mathrm{MHz}$

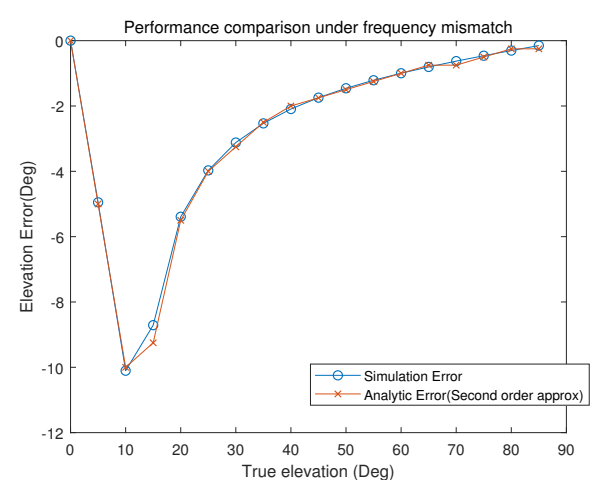

(b) RF: $1030 \mathrm{MHz}$

Figure 9. Analytically-derived elevation error and simulation-based error with respect to the true elevation of the incident signal $\left(f_{R F}=\frac{c}{\lambda_{R F}}=1020 \mathrm{MHz}\right.$ and $\left.1030 \mathrm{MHz}\right)$.

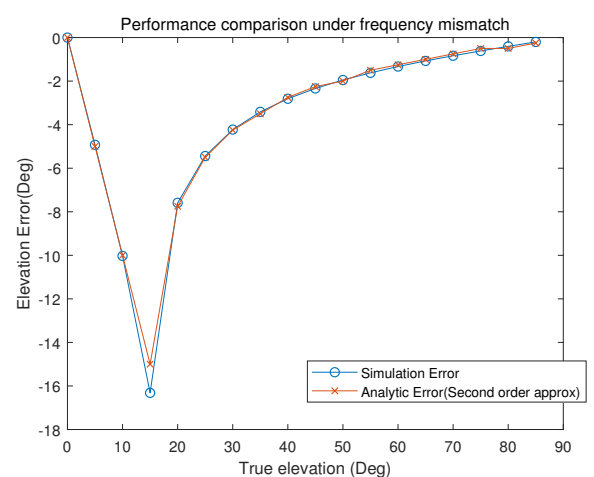

(a) RF: $1040 \mathrm{MHz}$

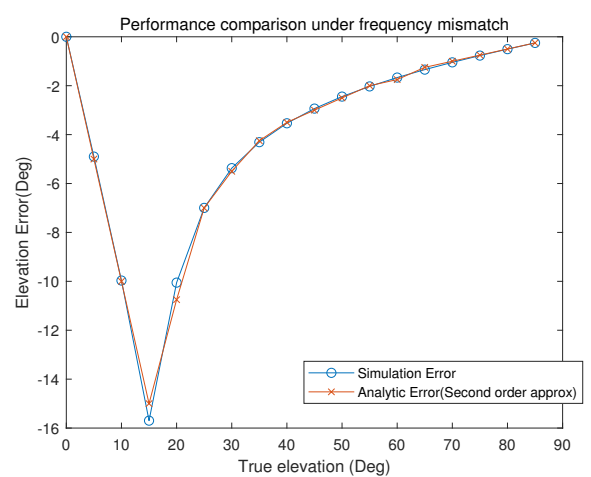

(b) RF: $1050 \mathrm{MHz}$

Figure 10. Analytically-derived elevation error and simulation-based error with respect to the true elevation of the incident signal $\left(f_{R F}=\frac{c}{\lambda_{R F}}=1040 \mathrm{MHz}\right.$ and $\left.1050 \mathrm{MHz}\right)$.

\section{Conclusions}

In this paper, for the interferometer DOA estimation algorithm, explicit expressions of the azimuth estimation error and elevation estimation error due to the frequency mismatch were derived and validated by numerical results.

The main contribution of this paper was to derive the azimuth and elevation error analytically when the frequency of the array manifold was different from the carrier frequency of the incident signal source. The derivation in this paper was verified by comparing the simulation-based estimation error with the analytically-derived estimation error.

It was derived that the analytic azimuth estimation error was identically zero even when the frequency of the incident signal was different from the nominal frequency used in the calculation of the array manifold, regardless of the true azimuth, $\theta^{(0)}$, and the true elevation, $\psi^{(0)}$. Since no approximation was used in deriving (26), the azimuth estimation error was exactly, not approximately, equal to zero even when the actual frequency of the incident signal was not equal to the nominal frequency employed in the calculation of the array manifold, which was confirmed in the upper plots of Figures 2 and 3 in the numerical results.

For the elevation estimation error, to improve the agreement between the analytically-derived results and the simulation-based results, $\cos (\Delta \psi) \approx 1-\frac{\Delta \psi^{2}}{2}, \sin (\Delta \psi) \approx \Delta \psi$, rather than $\cos (\Delta \psi) \approx 1$, $\sin (\Delta \psi) \approx \Delta \psi$, could be adopted. 
It was illustrated in the lower plots of Figures $2 \mathrm{~b}$ and $3 \mathrm{a}, \mathrm{b}$ that the analytically-derived elevation estimation errors based on the approximations of $\cos (\Delta \psi) \approx 1-\frac{\Delta \psi^{2}}{2}, \sin (\Delta \psi) \approx \Delta \psi$ showed better agreement with the simulation-based elevation estimation error than the analytically-derived elevation estimation errors based on the approximations of $\cos (\Delta \psi) \approx 1, \sin (\Delta \psi) \approx \Delta \psi$. For a given approximation, the agreement of the simulation-based results and the analytically-derived results improved with the decrease of $|\Delta \psi|$, which was confirmed by comparing the lower plots of Figures $2 \mathrm{~b}$ and $3 \mathrm{a}, \mathrm{b}$.

The performance due to the frequency mismatch reinforced once again the necessity of the wide-band DOA estimation algorithm. However, an interferometer using the narrow-band method may still be effective in the DOA of a signal source with a narrow bandwidth or a high elevation angle.

Author Contributions: C.-B.K. performed the MATLAB simulation and wrote the initial draft. J.-H.L. and C.-B.K. derived the mathematical formulation of the proposed scheme. In addition, J.-H.L. checked the numerical results and corrected the manuscript. All authors read and agreed to the published version of the manuscript.

Funding: This research was supported by the Basic Science Research Program through the National Research Foundation of Korea (NRF) funded by the Ministry of Education (2018R1D1A1B07048294). The authors gratefully acknowledge the support from the Electronic Warfare Research Center at Gwangju Institute of Science and Technology (GIST), originally funded by the Defense Acquisition Program Administration (DAPA) and the Agency for Defense Development (ADD).

Conflicts of Interest: The authors declare no conflict of interest.

Appendix A. Phase Difference between the Phase at the $m^{\text {th }}$ Sensor Location and the Phase at the Origin for an Incident Wave from $\theta=\theta^{(0)}$ and $\psi=\psi^{(0)}$ with a Wavelength of $\lambda_{R F}$

In this Appendix, the sensor location vector for a uniform circular array of a radius $r$ with $M$ sensors is given by:

$$
\mathbf{z}_{m}=\left[r \cos \left(\frac{2 \pi m}{M}\right), r \sin \left(\frac{2 \pi m}{M}\right), 0\right]^{T} .
$$

The direction vector for an incident signal from $\left(\theta^{(0)}, \psi^{(0)}\right)$ can be written as:

$$
\kappa=\left[\cos \left(\psi^{(0)}\right) \cos \left(\theta^{(0)}\right), \cos \left(\psi^{(0)}\right) \sin \left(\theta^{(0)}\right), \sin \left(\psi^{(0)}\right)\right]^{T} .
$$

The time delay between the $m^{\text {th }}$ sensor location and the origin can be written as:

$$
\tau(m)=\kappa^{T} \mathbf{z}_{m} / c
$$

The phase difference between the $m^{\text {th }}$ sensor location and the origin is given by the product of the angular frequency and time delay:

$$
\phi_{m}=\omega \times \text { Timedelay }=\omega \frac{\kappa^{T} \mathbf{z}_{m}}{c}=2 \pi \frac{\kappa^{T} \mathbf{z}_{m}}{\lambda}
$$

By substituting (A1) and (A2) in (A4), we have:

$$
\begin{gathered}
\varphi_{m}=\omega \times \text { Timedelay }=\omega \frac{\kappa^{T} \mathbf{z}_{m}}{c}=2 \pi \frac{\kappa^{T} \mathbf{z}_{m}}{\lambda} \\
=2 \pi \frac{r}{\lambda}\left[\cos \left(\frac{2 \pi m}{M}\right) \cos \left(\theta^{(0)}\right) \cos \left(\psi^{(0)}\right)+\sin \left(\frac{2 \pi m}{M}\right) \sin \left(\theta^{(0)}\right) \cos \left(\psi^{(0)}\right)\right] \\
=2 \pi \frac{r}{\lambda}\left[\cos \left(\theta^{(0)}-\frac{2 \pi m}{M}\right) \cos \left(\psi^{(0)}\right)\right]
\end{gathered}
$$


Appendix B. Proof of $\sum_{m=0}^{M-1} \cos ^{2}\left(a_{m}\right)=\sum_{m=0}^{M-1} \sin ^{2}\left(a_{m}\right)=\frac{M}{2}, \sum_{m=0}^{M-1} \sin \left(a_{m}\right) \cos \left(a_{m}\right)=0$

If $\quad \sum_{m=0}^{M-1} \exp \left(j\left(2 \theta^{(0)}-\frac{4 \pi m}{M}\right)\right)$ can be shown to be identically zero, $\sum_{m=0}^{M-1}\left(\cos \left(2 \theta^{(0)}-\frac{4 \pi m}{M}\right)+j \sin \left(2 \theta^{(0)}-\frac{4 \pi m}{M}\right)\right)$ is also equal to zero via the Euler formula.

Based on the summation of a geometric series, $\sum_{m=0}^{M-1} \exp \left(j\left(2 \theta^{(0)}-\frac{4 \pi m}{M}\right)\right)$ is equal to zero:

$$
\begin{aligned}
& \sum_{m=0}^{M-1} \exp \left(j \cdot\left(2 \theta^{(0)}-\frac{4 \pi m}{M}\right)\right)=\exp \left(j \cdot 2 \theta^{(0)}\right)\left(1+\exp \left(-j \cdot \frac{4 \pi}{M}\right)+\exp \left(-j \cdot \frac{8 \pi}{M}\right)+\cdots+\exp \left(-j \cdot \frac{4 \pi(M-1)}{M}\right)\right) \\
& =\exp \left(j \cdot 2 \theta^{(0)}\right)\left(\frac{1-\exp \left(-j \cdot \frac{4 \pi}{M} \cdot M\right)}{1-\exp \left(-j \cdot \frac{4 \pi}{M}\right)}\right)=0 .
\end{aligned}
$$

From (A6), since $\sum_{m=0}^{M-1} \exp j\left(2 a_{m}\right)$ is identically zero, we have:

$$
\begin{gathered}
\sum_{m=0}^{M-1}\left(\cos \left(2 a_{m}\right)+j \sin \left(2 a_{m}\right)\right)=0 \\
\sum_{m=0}^{M-1} \cos \left(2 a_{m}\right)+j \sum_{m=0}^{M-1} \sin \left(2 a_{m}\right)=0
\end{gathered}
$$

Note that $\cos \left(2 a_{m}\right)$ and $\sin \left(2 a_{m}\right)$ are real-valued for all $m$. Since the real number is closed under addition, $\sum_{m=0}^{M-1} \cos \left(2 a_{m}\right)$ is also real. Similarly, $\sum_{m=0}^{M-1} \sin \left(2 a_{m}\right)$ is also real. From (A8), both $\sum_{m=0}^{M-1} \cos \left(2 a_{m}\right)$ and $\sum_{m=0}^{M-1} \sin \left(2 a_{m}\right)$ should be equal to zero since $\sum_{m=0}^{M-1} \cos \left(2 a_{m}\right)$ and $\sum_{m=0}^{M-1} \sin \left(2 a_{m}\right)$ are real-valued:

$$
\begin{aligned}
& \sum_{m=0}^{M-1} \cos \left(2 a_{m}\right)=0 \\
& \sum_{m=0}^{M-1} \sin \left(2 a_{m}\right)=0 .
\end{aligned}
$$

Due to $\sum_{m=0}^{M-1} \cos \left(2 a_{m}\right)=\sum_{m=0}^{M-1}\left(\cos \left(2 \theta^{(0)}-\frac{4 \pi m}{M}\right)\right)=0 \quad$ and $\sum_{m=0}^{M-1} \sin \left(2 a_{m}\right)=$ $\sum_{m=0}^{M-1} \sin \left(2 \theta^{(0)}-\frac{4 \pi m}{M}\right)=0, \sum_{m=0}^{M-1} \cos ^{2}\left(a_{m}\right), \sum_{m=0}^{M-1} \sin ^{2}\left(a_{m}\right)$, and $\sum_{m=0}^{M-1} \sin \left(a_{m}\right) \cos \left(a_{m}\right)$ can be expressed as follows:

$$
\begin{gathered}
\sum_{m=0}^{M-1} \cos ^{2}\left(a_{m}\right)=\sum_{m=0}^{M-1}\left(\frac{1+\cos \left(2 a_{m}\right)}{2}\right)=\frac{M}{2}+\sum_{m=0}^{M-1} \cos \left(2 a_{m}\right)=\frac{M}{2} \\
\sum_{m=0}^{M-1} \sin ^{2}\left(a_{m}\right)=\sum_{m=0}^{M-1}\left(\frac{1-\cos \left(2 a_{m}\right)}{2}\right)=\frac{M}{2}-\sum_{m=0}^{M-1} \cos \left(2 a_{m}\right)=\frac{M}{2} \\
\sum_{m=0}^{M-1}\left(\sin \left(a_{m}\right) \cos \left(a_{m}\right)\right)=\frac{1}{2} \sum_{m=0}^{M-1} \sin \left(2 a_{m}\right)=0 .
\end{gathered}
$$




\section{References}

1. Abeywickrama, S.; Jayasinghe, L.; Fu, H.; Yuen, C. RF-based direction finding of UAVs using DNN. ArXiv 2017, arXiv:1712.01154.

2. Chang, X.; Yang, C.; Shi, X.; Li, P.; Shi, Z.; Chen, J. Feature extracted DOA estimation algorithm using acoustic array for drone surveillance. In Proceedings of the 2018 IEEE 87th Vehicular Technology Conference (VTC), Porto, Portugal, 3-6 June 2018; pp. 1-5.

3. Blair, W.D.; Watson, G.A.; Kirubarajan, T.; Bar-Shalom, Y. Benchmark for radar allocation and tracking in ECM. IEEE Trans. Aerosp. Electron. Syst. 1998, 34, 1097-1114. [CrossRef]

4. Zheng, Y.; Tseng, S.; Yu, K. Closed-form four-channel monopulse two-target resolution. IEEE Trans. Aerosp. Electron. Syst. 2003, 39, 1083-1089. [CrossRef]

5. Sharenson, S. Angle estimation accuracy with a monopulse radar in the search mode. IEEE Trans. Aerosp. Navig. Electron. 1962, 9, 175-179. [CrossRef]

6. Malioutov, D.; Cetin, M.; Willsky, A.S. A sparse signal reconstruction perspective for source localization with sensor arrays. IEEE Trans. Signal Process. 2005, 53, 3010-3022. [CrossRef]

7. Zheng, J.; Kaveh, M. Sparse spatial spectral estimation : A covariance fitting algorithm, performacne and regularization. IEEE Trans. Signal Process. 2013, 61, 2767-2777. [CrossRef]

8. Das, A.; Sejnowski, T.J. Narrowband and wideband off-grid direction of arrival estimation via sparse bayesian learning. IEEE J. Ocean. Eng. 2018. 43, 108-118. [CrossRef]

9. Kim, S.; Oh, D.; Lee, A.J. Joint DFT-ESPRIT estimation for TOA and DOA in vehicle FMCW Radars. IEEE Antennas Wirel. Propag. Lett. 2018, 43, 108-118. [CrossRef]

10. Stallings, W. Wireless Communications and Networks; Prentice Hall: Upper Saddle River, NJ, USA, 2004.

11. Kamel, A.; Abdelfettah, C.; Moussa, Y. Application of the technical Frequency-Hopped to the localization of sources by array of sensors and application to the algorithm Music. In Proceedings of the IEEE Sixth International Symposium on Spread Spectrum Techniques and Applications, Parsippany, NJ, USA, 6-8 September 2000; Volume 1, pp. 78-82.

12. Eric, M.; Dukic, M.L.; Obradovic, M. Frequency hopping signal separation by spatio-frequency analysis based on the MUSIC method. In Proceedings of the 2000 IEEE Sixth International Symposium on Spread Spectrum Techniques and Applications, ISSTA 2000, Parsippany, NJ, USA, 6-8 September 2000; Volume 1, pp. 78-82.

13. Aydin, L.; Polydoros, A. Joint hop-timing estimation for FH signals using a coarsely channelized receiver. Mil. Commun. Conf. 1995, 1, 769-773.

14. Wong, K.T. Blind beamforming/geolocation for wideband-FFH"s with unknown hop-sequences. IEEE Trans. Aerosp. Electron. Syst. 2001, 1, 65-76. [CrossRef]

15. Younger, J.P.; Reid, I.M. Interferometer AOA determination using precalculated phases. IEEE Radio Sci. 2017, 52, 1058-1066. [CrossRef]

16. Burtnyk, N.; McLeish, C.W.; Wolfe, J. Performance of an interferometer direction finder for the H.F. band. Proc. Inst. Electr. Eng. 1965, 112, 2055-2059. [CrossRef]

17. Li, C.; Xian-Ci, X. Performance study of 2-D DOA estimation using UCA with five sensors. In Proceedings of the IEEE 2002 International Conference on Communications, Circuits and Systems and West Sino Expositions, Chengdu, China, 29 June-1 July 2002; Volume 2, pp. 1012-1026.

18. Jiang, K.; Wu, Y.; Wu, X.; Cai, Y.; Zhou, J.; Du, L. A novel ambiguity resolution method of rolling Interferometer based on differential reduction. Radio Sci. 2007, 52, 1058-1066.

19. Liu, Z.; Guo, F. Azimuth and elevation estimation with rotating long-baseline interferometers. IEEE Trans. Signal Process. 2015, 63, 2405-2419. [CrossRef]

20. Moghaddasi, J.; Djerafi, T.; Wu, K. Multiport interferometer-enabled 2-D angle of arrival (AOA) estimation system. IEEE Trans. Microw. Theory Tech. 2017, 65, 1767-1779. [CrossRef]

21. Schmidt, R. Multiple emitter location and signal parameter estimation. IEEE Trans. Antennas Propag. 1986, 34, 276-280. [CrossRef]

22. Roy, R.; Kailath, T. ESPRIT-estimation of signal parameters via rotational invariance techniques. IEEE Trans. Acoust. Speech Signal Process. 1989, 37, 984-995. [CrossRef]

23. Balogh, L.; Kollar, I. Angle of arrival estimation based on interferometer principle. Proc. IEEE Int. Symp. Intell. Signal Process. 2003, 52, 1058-1066. 
24. Wei, H.; Wang, J.; Ye, S. An algorithm of estimation direction of arrival for phase interferometer array using cosine function. J. Electron. Inf. Technol. (Chin.) 2007, 29, 2665-2668.

25. An, X.; Feng, Z.; Wang, W.; Li, T. A single channel correlative interferometer direction finder using VXI receiver. In Proceedings of the International Conference on Microwave and Milimeter Wave Technology, Beijing, China, 17-19 August 2002; pp. 1158-1161.

26. Qin, L.; Jia, K.; He, Z. Performance analysis of correlative interferometer direction finder using cosine function. In Proceedings of the 2010 the 10th IEEE International Conference on Signal Processing ICSP2010, Beijing, China, 24-28 October 2010.

27. Wei, H.W.; Shi, Y.G. Performance analysis and comparison of correlative interferometers for direction finding. In Proceedings of the 2010 the 10th IEEE International Conference on Signal Processing ICSP2010, Beijing, China, 24-28 October 2010.

28. Wang, H.; Kaveh, M. Coherent signal-subspace processing for the detection and estimation of angles of arrival of multiple wideband sources. IEEE Trans. Acoust. Speech Signal Process. 1985, 33, 823-831. [CrossRef]

29. Hung, H.; Kaveh, M. Focusing matrices for coherent signal-subspace processing. IEEE Trans. Acoust. Speech Signal Process. 1988, 36, 1272-128. [CrossRef]

30. Di Claudio, E.D.; Parisi, R. WAVES: weighted average of signal subspaces for robust wideband direction finding. IEEE Trans. Signal Process. 2001, 49, 2179-2191. [CrossRef]

31. Lee, T.-S. Efficient wide-band source localization using beamforming invariance technique. IEEE Trans. Signal Process. 1994, 42, 1376-1387.

32. Yoon, Y.-S.; Kaplan, L.M.; McClellan, J.J. TOPS: New DOA estimator for wideband signals. IEEE Trans. Signal Process. 2006, 54, 1977-1989. [CrossRef]

(C) 2020 by the authors. Licensee MDPI, Basel, Switzerland. This article is an open access article distributed under the terms and conditions of the Creative Commons Attribution (CC BY) license (http:/ / creativecommons.org/licenses/by/4.0/). 